\title{
Banbury Forum Consensus Statement on the Path Forward for Digital Mental Health Treatment
}

David C. Mohr, PhD, ${ }^{1}$ Francisca Azocar, $\mathrm{PhD},{ }^{2}$ Andrew Bertagnolli, $\mathrm{PhD},{ }^{3}$ Tanzeem

Choudhury, PhD, ${ }^{4}$ Paul Chrisp, PhD,${ }^{5}$ Richard Frank, PhD, ${ }^{6}$ Henry Harbin, MD ${ }^{7}$ Trina Histon, $\mathrm{PhD},{ }^{8}$ Debra Kaysen, $\mathrm{PhD},{ }^{9},{ }^{10}$ Camille, Nebeker, $\mathrm{PhD},{ }^{11}$ Derek Richards, $\mathrm{PhD},{ }^{12,13}$ Stephen M. Schueller, PhD,${ }^{14}$ Nickolai Titov, PhD.,${ }^{15}$ John Torous, MD., ${ }^{16}$ Patricia A. Areán, $\mathrm{PhD},{ }^{17}$ on behalf of the Banbury Forum on Digital Mental Health

1. Department of Preventive Medicine, Center for Behavioral Intervention Technologies, Northwestern University, Chicago, IL

2. Optum, San Francisco, CA

3. One Medical and Alliant International University, San Francisco, CA

4. Information Science, Cornell Tech, New York, NY

5. National Institute for Health and Care Excellence

6. Department of Healthcare Policy, Harvard Medical School, Boston, MA

7. Magellan Health - Puyallup, WA

8. Kaiser Permanente, Care Management Institute, Oakland, CA

9. Department of Psychiatry and Behavioral Sciences, Stanford University, Palo Alto, CA

10. National Center for PTSD, Veterans Affairs Palo Alto Healthcare System, Palo Alto, California

11. Department of Family Medicine and Public Health, University of California, San Diego, CA

12. University of Dublin, Trinity College, School of Psychology, E-mental health research group, Dublin, Ireland

13. SilverCloud Health, Clinical Research \& Innovation, Boston, MA

14. Department of Psychological Science, University of California, Irvine, CA

15. MindSpot Clinic, Department of Psychology, Macquarie University, Sydney, Australia

16. Department of Psychiatry, Harvard Medical School, Boston, MA

17. Department of Psychiatry, University of Washington, Seattle, WA

\section{Corresponding Authors}

David C. Mohr, PhD

Department of Preventive Medicine

Center for Behavioral Intervention Technologies

Northwestern University

750 N. Lakeshore Dr., $10^{\text {th }}$ Floor

Chicago, IL 60611

Email: d-mohr@,northwestern.edu
Patricia A. Areán, PhD

Department of Psychiatry

UW ALACRITY Center

University of Washington

1959 NE Pacific Street

Seattle, WA 98125

email: parean@uw.edu

Word Count: 2974

\section{GROUP INFORMATION}

The Banbury Forum on Digital Mental Health was co-chaired by Patricia A. Areán, PhD (University of Washington) and David C. Mohr, PhD (Northwestern University), and included 
Francisca Azocar, $\mathrm{PhD}$ (Optum), Andrew Bertagnolli, $\mathrm{PhD}$ (One Medical and Alliant International University-San Francisco), Tanzeem Choudhury, PhD (Cornell University), Paul Chrisp, $\mathrm{PhD}$ (National Institute for Health and Care Excellence), Mark Cunningham-Hill (Northeast Business Group on Health), Mary Czerwinski, PhD (Microsoft Research), Lu Dong, $\mathrm{PhD}$ (RAND Corporation), Munmun De Choudhury, PhD (Georgia Institute of Technology), Richard Frank, PhD (Harvard Medical School), Henry Harbin, MD (Magellan Health), Charlie Hartwell (Bridge Builders Collaborative), Trina Histon, PhD (Kaiser Permanente), Tom Insel, MD (Mindstrong), Debra Kaysen, PhD (Stanford University), Neil Leibowitz, MD (Talkspace), Camille Nebeker, $\mathrm{PhD}$ (University of California, San Diego), Theresa Nguyen, (Mental Health America), Derek Richards, PhD (University of Dublin, Trinity College and SilverCloud Health), Phil Ritter, JD (Meadows Mental Health Policy Institute), Stephen Schueller, PhD (University of California, Irvine), Nick Titov, PhD (Mindspot Clinic, Macquarie University), John Torous, MD (Harvard Medical School). 


\begin{abstract}
A major obstacle to mental health treatment for many Americans is accessibility: the U.S. faces a shortage of mental health providers, resulting in federally-designated shortage areas. Digital mental health treatments (DMHT) are effective interventions for common mental disorders but have not been widely adopted by the American healthcare system. National and international expert stakeholders representing healthcare organizations, insurance and payers, employers, patients, research, policy, health economics, DMHT companies, and the investment community attended two Banbury Forum meetings to review the evidence for DMHTs, identify the challenges to successful and sustainable implementation, understand factors that have contributed to more successful implementation internationally, and develop recommendations. The Banbury Forum proposed the following recommendations: (1) Guided DMHTs should be offered to all patients experiencing common mental disorders; (2) DMHT products and services should be reimbursable to support integration into the American healthcare landscape; (3) An evidence standards framework should be developed to support decision makers in evaluating DMHTs.
\end{abstract}

\title{
Highlights
}

- Digital mental health treatments (DMHTs) have consistently demonstrated effectiveness for common mental disorders, yet they have not been broadly integrated into our healthcare system.

- The Banbury Forum, which included a broad range of national and international stakeholders, made three recommendations based on the evidence: 1) DMHTs should be broadly adopted in the U.S. healthcare system; 2) reimbursement mechanisms should be established to enable that adoption; and 3) An evidence standards framework for the evaluation of DMHTs should be developed.

- The integration of DMHTs into care pathways could improve the efficiency of mental health services and would extend effective treatment to many people with mental health problems who are currently unable to access treatment.

\section{INTRODUCTION}

Each year, nearly $20 \%$ of Americans experience a diagnosable mental health condition. ${ }^{1}$ Many people experience barriers to care. ${ }^{2-5}$ The U.S. has a shortage of mental healthcare specialists, with nearly 120 million Americans living in federally designated mental health provider shortage areas. $^{6,7}$ The recent COVID-19 pandemic, which has increased the incidence of mental health problems, has further highlighted the challenges of mental health care access. ${ }^{8}$ Tele-mentalhealth has been expanding for more than a decade to overcome regional disparities ${ }^{9}$, and is now expanded more broadly under COVID- $19^{10}$ with calls to make this expansion permanent. ${ }^{11}$ Although this expansion reduces regional challenges in access to care, it does not address the 
overall lack of mental health providers in the US.

Digital Mental Health Treatments (DMHTs; apps and internet based care) could overcome both access and provider shortage problems. ${ }^{12}$ DMHTs are delivered remotely and have demonstrated effectiveness in more than 100 randomized controlled trials (RCTs). ${ }^{13-15}$ While DMHTs have begun to be integrated into healthcare systems in Europe and Australia, ${ }^{16-20}$ they have not been well integrated into the U.S. healthcare system.

The Banbury Forum for Digital Mental Health Treatment was formed to uncover the reasons why DMHTs have not been broadly adopted by the U.S. Healthcare system and to provide recommendations to overcome those challenges.

\section{PROCESS AND METHODS}

The Banbury Center convened a meeting of 23 international leaders and stakeholders representing healthcare organizations, insurance and payers, employers, patients, research, policy, health economics, DMHT companies, and the investment community, led by co-chairs Drs. Areán and Mohr. The forum met in October 2019 to review the current state of the evidence and identify the primary challenges to adoption of DMHTs in the American healthcare system, and to make recommendations to facilitate the successful and sustainable implementation of effective digital mental health interventions in the American healthcare system. Each stakeholder group identified core challenges and opportunities in the US. The forum reconvened remotely on June 2020 in response to the challenges COVID-19 raised for this report, adding new representatives with expertise in the pandemic. An initial draft of this consensus report was discussed and refined in light of the current challenges in accessing mental health services during the COVID-19 pandemic.

\section{RESULTS}

\section{Definition}

DMHTs support patients and clinicians in managing mental health using smartphone and web applications, with growing research investigating therapeutic videogames, virtual reality, and conversational agents. ${ }^{12}$ While all DMHTs are patient-facing, the degree to which a provider is part of the platform varies on a continuum from adjunctive (e.g., to support psychotherapy), to guided (key aspects of care are delivered by the technology, supported by a clinician, coach, or peer, who provides low-intensity support), to fully automated (used without human support, such as apps available from the app stores). ${ }^{21}$

\section{Opportunities}

There was broad consensus that DMHTs provide an effective and scalable method of extending the reach of effective mental health care. This was based on two areas of evidence, effectiveness and cost-effectiveness.

Effectiveness. Meta-analyses of at least 66 well-designed RCTs have demonstrated that guided DMHT is effective for common mental health conditions such as depression and anxiety disorders, comparable to standard face-to-face therapies. ${ }^{13,22,23}$ A meta-analysis of 8 RCTs has 
shown effectiveness of DMHTs for post-traumatic stress disorder (PTSD). ${ }^{24}$ DMHTs for depression and anxiety are effective at the mild, moderate, and severe ranges of symptom severity, ${ }^{25,26}$ and can be effective across the lifespan, with a growing number of RCTs demonstrating effectiveness in children and adolescents, ${ }^{27}$ and older adults. ${ }^{28,29}$

DMHTs are also effective for common problems associated with mental health, such as insomnia. ${ }^{30}$ Several RCTs of guided digital treatments for alcohol and substance abuse have shown significant but more modest benefits. ${ }^{31,32}$ Research on DMHTs for severe mental illness such as bipolar and psychotic disorders have shown feasibility, although efficacy data remains more limited..$^{33,34}$

Automated vs. Guided vs. Adjunctive DMHTs. Guided DMHTs, which include low intensity support from a clinician or coach via messaging or telephone, produce much larger benefits than fully automated DMHTs. ${ }^{22}$ Typically this support aims to maintain patient adherence to the app and monitor progress through periodic symptom assessment, but may also include assisting the patient in understanding concepts or skills training, and triaging patients who do not respond to the DMHT to a higher level of care. ${ }^{35}$

People can benefit from fully automated, unguided DMHTs, ${ }^{36}$ however, at a population-level they show small benefits, likely because dropout rates are higher in unguided than guided interventions. ${ }^{37,38}$ Only a few studies have examined the use of DMHT tools as adjunctive support for psychotherapy. While these have found that patients improve in treatments that blend DMHT with face-to-face psychotherapy, it is unclear whether the addition of DMHT tools results in any greater improvement or reduction in the amount of time required in psychotherapy. ${ }^{39,40}$

Cost-effectiveness. Emerging evidence supports the cost-effectiveness of guided DMHTs. ${ }^{20,41}$ Guided DMHTs can treat common mental health conditions with substantially less provider time than is typically required for standard psychotherapy. For example, stepped care programs that initiate treatment with a DMHT and move non-responders to traditionally delivered psychotherapy use about half the clinician time required for programs that do not have a stepped care option and use psychotherapy only. ${ }^{42}$ In addition, there is no difference in outcomes for DMHTs supported by non-mental health specialists compared to DMHTs supported by mental health specialists, ${ }^{43}$ which allows for task sharing options at lower levels of licensure and cost.

While some guided DMHTs met cost-effectiveness criteria, automated DMHTs typically have not, in part because their effectiveness is lower. ${ }^{41}$ However, whether to use fully automated or guided DMHTs remains controversial. The ratio of fixed costs (e.g. costs of EHR integration, licensing), required for both guided and automated DMHTs, to number of patient users decreases as a DMHT is scaled up. However, variable costs (e.g. therapists or care managers providing guidance), primarily associated with guided DMHTs, increase with each new patient. Indeed, even though automated DMHTs are less effective than guided, automated DMHT have been shown to cost less overall. ${ }^{44}$

\section{Challenges}

Around the globe there has been an increased in the adoption of DMHTs, owing to their 
effectiveness and potential to improve efficiency of mental healthcare delivery. For example, the English National Institute for Health and Care Excellence (NICE), which issues guidelines for clinical practice and health technologies in their National Health Service (NHS), approved the use of iCBT for depression and anxiety in mental health services in $2006 .{ }^{16}$ In 2009 the recommendation for the use of DMHTs was integrated into the guidelines for treatment of depression. ${ }^{17}$ In Australia, the federal government has funded a number of initiatives to deliver DMHTs for common mental health problems since at least 2010, such as MindSpot. ${ }^{45}$ In the Netherlands, insurance companies are required to cover payments for DMHTs and the government provides funding for the delivery of DMHTs, free of charge, for those who do not want to use treatments offered through usual care venues.

Reimbursement of DMHTs. A primary challenge in reimbursement of DMHTs is in the variability of payment methods that exist in the US. Some healthcare providing organizations such as Kaiser Permanente or the Veterans Health Administration operate completely outside the fee-for-service model. This creates greater flexibility in decisions around the adoption of new treatments and technologies but imposes strict budget constraints. For instance, Kaiser Permanente has begun offering DMHTs and views them as clinical tools and services that are part of a new standard of care. Some states, such as California, are also piloting the use of DMHTs in public mental health. Self-insured employers are adopting DMHTs as part of their service packages for employees. However, the vast majority of Americans are served by healthcare organizations that operate with a mix of payment models. In 2018, approximately $36 \%$ of healthcare payments were tied to bundled payments, shared saving, and other alternative payment methods (APMs), and the remainder were fee-for-service (some tied to value and quality and some not). ${ }^{46}$ APMs focus on improving outcomes and reducing costs, which could favor effective deployment of DMHTs. While the United States is gradually adopting APMs, the fee-for-service model is likely to remain a dominant form of payment for the foreseeable future. Fee-for-service relies on Current Procedural Terminology (CPT) codes or the Healthcare Common Procedure Coding System (HCPCS) to bill for services.

Currently there are no billing codes for DMHTs, making broad adoption of DMHT services financially unworkable in American healthcare organizations. ${ }^{47}$ However, in response to social distancing measures during the COVID-19 pandemic, the entire American healthcare system has made a remarkably rapid transition to remote care by relaxing the rules around telemedicine. ${ }^{48}$ The American Medical Association has an advisory group working on similar coverage issues for digital health tools. ${ }^{49}$ Recently, new CPT codes 98970-98792 were opened for online digital evaluation and management services. ${ }^{50}$ While these are only for physicians, physician assistants, and nurses, they could be expanded to cover a broader range of practitioners, who would support DMHTs. Reimbursement for the cost of DMHT products will also be required, and could be handled in a number of ways, including through device codes and/or embedding the cost of the product in the CPT code.

DMHT Evidence Standards Framework. An evidence standards framework is needed to support "digital formularies," which allow provider organizations and payers to identify preferred products. ${ }^{51}$ While the FDA does not currently enforce regulatory requirements on the kinds of software and functionality used by most DMHT tools, some companies have elected nonetheless to seek FDA clearance. However, there was consensus among relevant forum 
participants that FDA clearance, which focuses on safety and minimal effectiveness thresholds, does not provide adequate information for decision makers. The United States does not have a body such as England's NICE that evaluates effectiveness and cost-effectiveness of services and treatment, ${ }^{52}$ however this role is sometimes filled by non-governmental organizations such as the Institute for Clinical and Economic Review.

An evidence standards framework should integrate core ethical principles of respect for persons, beneficence and justice, ${ }^{53}$ must be specific enough to protect stakeholders, including patients, families, providers, and payers, and also being flexible enough to be applied to new forms of DMHTs that harness the affordances of technological advancements. A number of exemplar frameworks exist, which cover core principles for DMHT standards, ${ }^{52,54,55}$ upon which we elaborate below.

Benefit/Efficacy: The best practice standard for effectiveness consists of at least one wellpowered, well-designed RCT, conducted in a relevant setting, using an accepted conditionspecific clinical outcome with participants who are representative of the target population. This level of evidence should be required when a DMHT represents a novel intervention or new technological medium.

In the absence of data for a given tool, a minimum evidence standard may be applied if the DMHT is based on previously validated DMHT method. For instance, because there is a strong evidence base for guided internet based cognitive behavioral therapy (iCBT), ${ }^{37}$ a new iCBT product might be considered evidence-based if it has fidelity to core elements of iCBT platforms for example by meta-analysis of previous trials. For iCBT, very few of the products that claim to be CBT-based actually contain the core elements of CBT. ${ }^{56}$ When indirect scientific evidence might suffice, some evidence through non-experimental studies, such as single arm pilot studies with an appropriate sample of participants, should be conducted to evaluate the feasibility, acceptability, and safety of a platform. ${ }^{56-58}$

Engagement. Patient engagement has been a challenge for some DMHTs. While some trials have shown strong patient engagement in healthcare settings, ${ }^{59}$ others have had high dropout rates. ${ }^{60,61}$ Challenges in maintaining the engagement of healthcare workers tasked with coaching guided DMHTs can occur from difficulty fitting tasks into the workflow, lack of adequate training and support, and reluctance to taking on new tasks with unclear productivity metrics. ${ }^{62}$

A best practice standard would demonstrate high levels of sustained patient engagement with the tools in a well-designed RCT. Because provider engagement from RCTs may be difficult to generalize, as providers often work closely with research staff in trials, an evaluation of the user experience, including how useful it is, ease of use, efficiency, and satisfaction may be conducted with representative providers. ${ }^{63}$ Minimal standards for user engagement may include evidence that representatives from intended user groups were involved in the design and testing of the DMHT, and may also include user experience evaluations with representative users.

Data Sharing (Interoperability). DMHTs involve platforms that share data seamlessly among electronic health records, DMHT tools, and community-based sources for comprehensive population health management. Guided DMHTs should collect relevant data from the patient, 
which should be delivered to the provider to inform care. In most cases, this should include validated self-reported symptom severity. This is a critical component for measurement-based care, allowing the provider to monitor improvement and to intervene and triage to a higher level of care. ${ }^{64}$ It is also useful for providers to be able to see usage of the technology, to support patient engagement. ${ }^{35,65}$ Thus, DMHTs need requirements for data interoperability that support their intended aims and align with the larger movement in healthcare. ${ }^{66}$

Risk management. While there is no evidence from trials that DMHTs themselves are harmful or pose a risk, ${ }^{67}$ some DMHTs have been be found to provide advice that is potentially harmful. ${ }^{68}$ Mental health conditions also can increase risks, most notably suicide. ${ }^{69}$ Standards should include a careful review to ensure there is no content that is potentially harmful. DMHTs should include functionality that supports the identification and management of suicidality or other relevant risk factors.

Data Security and Privacy. Evidence standards should ensure that all data collected is kept confidential. While some companies have not been transparent about the use, sharing, or sale of data ${ }^{70}$ vendors also can have interests in using the data for a variety of reasonable purposes, including continuous improvement of the product. Privacy policies should be available to the patient, which explain data management processes, including what data is being stored, where it is stored, how it is stored, how long it will be stored, who has access to the data, and the purposes for which the data will be used.

Equity. In addition to the evidence standards described above, the forum also felt that issues addressing equity should be considered, including access barriers due to income, language or disability. The Americans with Disabilities Act directs most healthcare settings within the U.S. to ensure that patients who are blind, deaf-blind or visually impaired have equal access to participate in and benefit from all the goods and services provided by the healthcare facility. Standards should ensure that screen readers can parse content on a page to make DMHTs usable for blind populations. Several healthcare agencies have recommended that the readability of English language patient education materials should not be higher than fifth- to eighth-grade level, ${ }^{71}$ and services and patient materials should be provided in the patient's preferred language. ${ }^{72}$ Low income patients may have more tenuous connectivity, with limited Wi-Fi access and data plans, resulting in additional costs to the patient and higher rates of suspended service. ${ }^{73}$ Thus standards should consider the data requirements of a DMHT, and healthcare providing organizations should consider DMHT implementation plans that mitigate the potential barriers to low-income patients and patients with limited English proficiency.

\section{Recommendations}

The Banbury Forum was unanimous in making the following actionable recommendations:

Recommendation 1. Guided DMHTs should be offered as a treatment option to all patients experiencing depression, anxiety disorders, and PTSD.

There is a large evidence base, which has consistently shown that guided DMHTs are effective across the lifespan and for all levels of symptom severity. DMHTs should be integrated into care pathways to increase access to mental health treatment and used to optimize the 
efficiency of mental health services.

Recommendation 2. DMHT products and services should be reimbursable to support integration into the American healthcare landscape.

Absence of reimbursement mechanisms is the primary impediment for implementation of DMHTs in many healthcare organizations. DMHT reimbursement must cover both the cost of the DMHT product as well as the provider time at rates that are equal to reimbursement rates for similar amounts of time spent in face-to-face treatments. Reimbursement mechanisms must be integrated into the variety of reimbursement systems used through federal, state, and commercial payers.

Recommendation 3. An evidence standards framework should be developed to support digital formularies and decision making in healthcare organizations, states, and commercial health plans and payers in selecting DMHT products that are effective, usable, safe, and equitable.

While the US does not have a centralized process for creating evidence-based standards frameworks, these can be developed through non-governmental or professional organizations.

\section{DISCUSSION \& CONCLUSIONS}

There is a growing recognition that the United States requires a more sustainable approach to ensuring affordable access to effective mental healthcare delivery, including expansion of access, remotely delivered mental health services, and increased adoption of measurement based care. ${ }^{74}$ DMHTs are an effective method of delivering mental healthcare remotely, and collect outcomes that support measurement based care. Integration of DMHTs into care pathways would improve the efficiency of mental health services, for example through stepped care models where patients initiate treatment with lower cost DMHTs, preserving mental health specialist time for those who do not show sufficient response. ${ }^{42}$

The forum participants recognized that these recommendations, while necessary, are not sufficient to achieve integration of DMHTs into our healthcare system. Opening reimbursement mechanisms such as CPT and device codes does not guarantee that they will be used, as shown by the recent behavioral health integration codes, intended to support collaborative care. Using these codes requires substantial procedural and billing workflow adjustments that can be complicated for healthcare organization to implement. ${ }^{75}$ Co-payments can reduce patient uptake. Healthcare organizations will also require guidance on the integration of DMHTs into their care pathways. Clincians who support patients in their treatment through DMHTs will require training to obtain optimal engagement and outcomes. DMHT integration has an additional challenge, not encountered with collaborative care, as it relies on devices and connectivity that are not equally distributed across the American population. Device codes exist that could potentially enable purchasing devices and connectivity, and infrastructure is available, such as the Federal Lifeline Assistance program (a.k.a Obama Phones). However, as there have been few efforts on the part of healthcare organizations to assist low income patients in acquiring phones, tablets, computers, or data plans, policies and procedures will need to be developed to ensure patients are aware of these opportunities to improve connectivity.

The experience with collaborative care may offer some guidance on strategies to overcome these 
challenges. The University of Washington's Advanced Integrated Mental Health Solutions (AIMS) Center has provided a leadership role in advancing collaborative care, and more recently in the implementation of these codes. The AIMS Center as part of training in the implementation of effective collaborative care, also provides training and implementation assistance to healthcare organizations in developing procedures for reimbursement. A similar center could play an important role in supporting the effective adoption of DMHTs, including the use of CPT and device codes, definition of evidence standards for DMHTs, and how to make patients aware of opportunities to be better connected through technology. Implementation may also be facilitated by DMHT companies, which have an interest in supporting healthcare staff in effective implementation as well as healthcare organizations in developing efficient processes for reimbursement.

The United States has lagged behind other countries in the integration of digital mental health into the American healthcare system. While there is growing interest in adopting DMHTs, the absence of reimbursement mechanisms remains a primary obstacle to broad adoption. These recommendations are consistent with recent policy statements from Mental Health America, our nation's largest patient advocacy organization, calling for reimbursement of DMHTs. ${ }^{76}$ The American healthcare system has made a remarkably rapid transition to remote care by relaxing the rules around telemedicine ${ }^{48}$ and has begun considering codes that would support digital health administered by physicians and nurses. ${ }^{50}$ The need and momentum for the integration of DMHTs into our healthcare system are here. Enabling reimbursement would allow healthcare organizations to make DMHTs broadly available, with evidence standards that would support the selection of DMHT products and services that are effective and can be sustainably implemented.

\section{FUNDING}

The Banbury Center Forum on Digital Mental Health Treatment was funded by the Cold Spring Harbor Laboratory Corporate Sponsor Program, with additional support from Microsoft Corporation. 


\section{REFERENCES}

1. Substance Abuse and Mental Health Services Administration (SAMHSA). Results from the 2017 National Survey on Drug Use and Health. Substance Abuse and Mental Health Services Administration (SAMHSA). https://www.samhsa.gov/data/sites/default/files/cbhsqreports/NSDUHDetailedTabs2017/NSDUHDetailedTabs2017.htm\#ftn1. Published 2017. Accessed2019.

2. Thornicroft G, Chatterji S, Evans-Lacko S, et al. Undertreatment of people with major depressive disorder in 21 countries. Br J Psychiatry. 2017;210(2):119-124.

3. Alonso J, Liu Z, Evans-Lacko S, et al. Treatment gap for anxiety disorders is global: Results of the World Mental Health Surveys in 21 countries. Depress Anxiety. 2018;35(3):195-208.

4. Mohr DC, Ho J, Duffecy J, et al. Perceived barriers to psychological treatments and their relationship to depression. J Clin Psychol. 2010;66(4):394-409.

5. Mohr DC, Hart SL, Howard I, et al. Barriers to psychotherapy among depressed and nondepressed primary care patients. Ann Behav Med. 2006;32(3):254-258.

6. Bureau of Health Workforce Health Resources and Services Administration (HRSA). Designated Health Professional Shortage Areas Statistics. data.HRSA.gov: U.S. Department of Health \& Human Services;2020.

7. Kazdin AE, Blase SL. Rebooting Psychotherapy Research and Practice to Reduce the Burden of Mental Illness. Perspectives on Psychological Science. 2011;6(1):21-37.

8. Twenge JM, Joiner TE. Mental distress among U.S. adults during the COVID-19 pandemix. PsyArXiv Preprints. 2020.

9. Mehrotra A, Huskamp HA, Souza J, et al. Rapid Growth In Mental Health Telemedicine Use Among Rural Medicare Beneficiaries, Wide Variation Across States. Health Aff (Millwood). 2017;36(5):909-917.

10. Federation of State Medical Boards. U.S. states and territories modifying requirements for telehealth in response to COVID-19. Federation of State Medical Boards. https://www.fsmb.org/siteassets/advocacy/pdf/states-waiving-licensure-requirements-fortelehealth-in-response-to-covid-19.pdf. Published 2020. Accessed June 10, 2020, 2020.

11. American Psychiatric Association. APA to CMS: Some telehealth changes made during COVID19 should become permanent. American Psychiatric Association.

http://alert.psychnews.org/2020/06/apa-to-cms-some-telehealth-changes-made.html. Published 2020. Accessed June 10, 2020, 2020.

12. Arean PA, Leshner A, Barch DM, et al. Opportunities and Challenges of Developing Information Technologies on Behavioral and Social Science Clinical Research. 2017. https://www.nimh.nih.gov/about/advisory-boards-and-groups/namhc/reports/opportunities-andchallenges-of-developing-information-technologies-on-behavioral-and-social-science-clinicalresearch.shtml.

13. Andrews G, Basu A, Cuijpers P, et al. Computer therapy for the anxiety and depression disorders is effective, acceptable and practical health care: An updated meta-analysis. $J$ Anxiety Disord. 2018;55:70-78.

14. Firth J, Torous J, Nicholas J, et al. The efficacy of smartphone-based mental health interventions for depressive symptoms: a meta-analysis of randomized controlled trials. World Psychiatry. 2017;16(3):287-298.

15. Firth J, Torous J, Nicholas J, Carney R, Rosenbaum S, Sarris J. Can smartphone mental health interventions reduce symptoms of anxiety? A meta-analysis of randomized controlled trials. $J$ Affect Disord. 2017;218:15-22.

16. National Institute for Health and Clinical Excellence (NICE). Computerised cognitive behaviour therapy for depression and anxiety. London: National Institute for Health and Clinical Excellence (NICE);2006.

17. National Institute for Health and Care Excellence. The treatment and management of depression in adults. London, UK: National National Institute for Clinical Excellence;2009. 
18. Titov N, Dear BF, Staples LG, et al. The first 30 months of the MindSpot Clinic: Evaluation of a national e-mental health service against project objectives. Aust N Z J Psychiatry.

2017;51(12):1227-1239.

19. Duffy D, Enrique A, Connell S, Connolly C, Richards D. Internet-Delivered Cognitive Behavior Therapy as a Prequel to Face-To-Face Therapy for Depression and Anxiety: A Naturalistic Observation. Front Psychiatry. 2019;10:902.

20. Richards D, Enrique A, Eilert N, et al. A pragmatic randomized waitlist-controlled effectiveness and cost-effectiveness trial of digital interventions for depression and anxiety. NPJ Digit Med. 2020;3:85.

21. Hermes ED, Lyon AR, Schueller SM, Glass JE. Measuring the Implementation of Behavioral Intervention Technologies: Recharacterization of Established Outcomes. J Med Internet Res. 2019;21(1):e11752.

22. Linardon J, Cuijpers P, Carlbring P, Messer M, Fuller-Tyszkiewicz M. The efficacy of appsupported smartphone interventions for mental health problems: a meta-analysis of randomized controlled trials. World Psychiatry. 2019;18(3):325-336.

23. Andersson G, Cuijpers P, Carlbring P, Riper H, Hedman E. Guided Internet-based vs. face-toface cognitive behavior therapy for psychiatric and somatic disorders: a systematic review and meta-analysis. World Psychiatry. 2014;13(3):288-295.

24. Olthuis JV, Wozney L, Asmundson GJ, Cramm H, Lingley-Pottie P, McGrath PJ. Distancedelivered interventions for PTSD: A systematic review and meta-analysis. $J$ Anxiety Disord. 2016;44:9-26.

25. Bower P, Kontopantelis E, Sutton A, et al. Influence of initial severity of depression on effectiveness of low intensity interventions: meta-analysis of individual patient data. $B M J$. 2013;346:f540.

26. Wright JH, Mishkind M, Eells TD, Chan SR. Computer-Assisted Cognitive-Behavior Therapy and Mobile Apps for Depression and Anxiety. Curr Psychiatry Rep. 2019;21(7):62.

27. Ebert DD, Zarski AC, Christensen H, et al. Internet and computer-based cognitive behavioral therapy for anxiety and depression in youth: a meta-analysis of randomized controlled outcome trials. PLoS One. 2015;10(3):e0119895.

28. Titov N, Dear BF, Ali S, et al. Clinical and cost-effectiveness of therapist-guided internetdelivered cognitive behavior therapy for older adults with symptoms of depression: a randomized controlled trial. Behav Ther. 2015;46(2):193-205.

29. Silfvernagel K, Westlinder A, Andersson S, et al. Individually tailored internet-based cognitive behaviour therapy for older adults with anxiety and depression: a randomised controlled trial. Cogn Behav Ther. 2018;47(4):286-300.

30. Zachariae R, Lyby MS, Ritterband LM, O'Toole MS. Efficacy of internet-delivered cognitivebehavioral therapy for insomnia - A systematic review and meta-analysis of randomized controlled trials. Sleep Med Rev. 2016;30:1-10.

31. Riper H, Blankers M, Hadiwijaya $\mathrm{H}$, et al. Effectiveness of guided and unguided low-intensity internet interventions for adult alcohol misuse: a meta-analysis. PLoS One. 2014;9(6):e99912.

32. Boumparis N, Karyotaki E, Schaub MP, Cuijpers P, Riper H. Internet interventions for adult illicit substance users: a meta-analysis. Addiction. 2017;112(9):1521-1532.

33. Firth J, Torous J. Smartphone Apps for Schizophrenia: A Systematic Review. JMIR Mhealth Uhealth. 2015;3(4):e102.

34. Gliddon E, Barnes SJ, Murray G, Michalak EE. Online and mobile technologies for selfmanagement in bipolar disorder: A systematic review. Psychiatr Rehabil J. 2017;40(3):309-319.

35. Schueller SM, Tomasino KN, Mohr DC. Integrating Human Support into Behavioral Intervention Technologies: The Efficiency Model of Support. Clinical Psychology: Science and Practice. 2016(24):27-45. 
36. Mohr DC, Schueller SM, Tomasino KN, et al. Comparison of the Effects of Coaching and Receipt of App Recommendations on Depression, Anxiety, and Engagement in the IntelliCare Platform: Factorial Randomized Controlled Trial. J Med Internet Res. 2019;21(8):e13609.

37. Karyotaki E, Riper H, Twisk J, et al. Efficacy of Self-guided Internet-Based Cognitive Behavioral Therapy in the Treatment of Depressive Symptoms: A Meta-analysis of Individual Participant Data. JAMA Psychiatry. 2017;74(4):351-359.

38. Weisel KK, Fuhrmann LM, Berking M, Baumeister H, Cuijpers P, Ebert DD. Standalone smartphone apps for mental health-a systematic review and meta-analysis. NPJ Digit Med. 2019;2:118.

39. Kenter RF, van de Ven PM, Cuijpers P, et al. Costs and effects of Internet cognitive behavioral treatment blended with face-to-face treatment: Results from a naturalistic study. Internet Interventions. 2015;2(1):77-83.

40. Wright JH, Wright AS, Albano AM, et al. Computer-assisted cognitive therapy for depression: maintaining efficacy while reducing therapist time. Am J Psychiatry. 2005;162(6):1158-1164.

41. Paganini S, Teigelkotter W, Buntrock C, Baumeister H. Economic evaluations of internet- and mobile-based interventions for the treatment and prevention of depression: A systematic review. $J$ Affect Disord. 2018;225:733-755.

42. Mohr DC, Lattie EG, Tomasino KN, et al. A randomized noninferiority trial evaluating remotelydelivered stepped care for depression using internet cognitive behavioral therapy (CBT) and telephone CBT. Behav Res Ther. 2019;123:103485.

43. Titov N, Andrews G, Davies M, McIntyre K, Robinson E, Solley K. Internet treatment for depression: a randomized controlled trial comparing clinician vs. technician assistance. PLoS One. 2010;5(6):e10939.

44. Abhulimen S, Hirsch A. Quantifying the economic impact of a digital self-care behavioral health platform on Missouri Medicaid expenditures. J Med Econ. 2018;21(11):1084-1090.

45. Lee YC, Gao L, Dear BF, Titov N, Mihalopoulos C. The Cost-effectiveness of the Online MindSpot Clinic for the Treatment of Depression and Anxiety in Australia. J Ment Health Policy Econ. 2017;20(4):155-166.

46. Health Care Payment Learning \& Action Network. APM Measurement: Progress of alternative payment models. Health Care Payment Learning \& Action Network;2019.

47. Powell AC, Bowman MB, Harbin HT. Reimbursement of Apps for Mental Health: Findings From Interviews. JMIR Ment Health. 2019;6(8):e14724.

48. Nouri S, Choong EC, Lyles CR, Karliner L. Addressing equity in telemedicine for chronic disease management during the Covid-19 pandemic. NEJM Catalyst. 2020. https://catalyst.nejm.org/doi/full/10.1056/CAT.20.0123\#r2. Published May 4, 2020. Accessed June 10, 2020.

49. American Medical Association. Digital Medicine Payment Advisory Group. American Medical Association. https://www.ama-assn.org/practice-management/digital/digital-medicine-paymentadvisory-group. Published 2020. Accessed June 24, 2020, 2020.

50. Nicoletti B. CPT ${ }^{\circledR}$ codes (99421-99423) - and payment for - online digital evaluation and management (E/M) services. CodingIntel. https://codingintel.com/cpt-codes-online-digitalevaluation-and-management-services/. Published 2020. Accessed July 13, 2020.

51. Gordon WJ, Landman A, Zhang H, Bates DW. Beyond validation: getting health apps into clinical practice. NPJ Digit Med. 2020;3:14.

52. National Institute for Health and Care Excellence. Evidence standards framework for digital health technologies. London, UK: National Institute for Clinical Excellence;2019.

53. Nebeker C, Bartlett Ellis RJ, Torous J. Development of a decision-making checklist tool to support technology selection in digital health research. Transl Behav Med. 2019.

54. Carlo AD, Hosseini Ghomi R, Renn BN, Arean PA. By the numbers: ratings and utilization of behavioral health mobile applications. NPJ Digit Med. 2019;2:54. 
55. Torous JB, Chan SR, Gipson SYT, et al. A Hierarchical Framework for Evaluation and Informed Decision Making Regarding Smartphone Apps for Clinical Care. Psychiatr Serv. 2018;69(5):498500.

56. Huguet A, Rao S, McGrath PJ, et al. A Systematic Review of Cognitive Behavioral Therapy and Behavioral Activation Apps for Depression. PLoS One. 2016;11(5):e0154248.

57. Andersson G. Internet interventions: Past, present and future. Internet Interv. 2018;12:181-188.

58. Richards D, Richardson T. Computer-based psychological treatments for depression: a systematic review and meta-analysis. Clin Psychol Rev. 2012;32(4):329-342.

59. Graham AK, Greene CJ, Kwasny MJ, et al. Coached Mobile App Platform for the Treatment of Depression and Anxiety Among Primary Care Patients: A Randomized Clinical Trial. JAMA Psychiatry. 2020.

60. Gilbody S, Brabyn S, Lovell K, et al. Telephone-supported computerised cognitive-behavioural therapy: REEACT-2 large-scale pragmatic randomised controlled trial. Br J Psychiatry. 2017.

61. Gilbody S, Littlewood E, Hewitt C, et al. Computerised cognitive behaviour therapy (cCBT) as treatment for depression in primary care (REEACT trial): large scale pragmatic randomised controlled trial. BMJ. 2015;351:h5627.

62. Bertagnoli A. Digital mental health: Challenges in Implementation. American Psychiatric Association; 2018; New York, NY.

63. Nielsen J. Usability inspection methods. New York: John Wiley \& Sons; 1994.

64. Harding KJ, Rush AJ, Arbuckle M, Trivedi MH, Pincus HA. Measurement-based care in psychiatric practice: a policy framework for implementation. J Clin Psychiatry. 2011;72(8):11361143.

65. Mohr DC, Cuijpers P, Lehman K. Supportive Accountability: A Model for Providing Human Support to Enhance Adherence to eHealth Interventions. J Med Internet Res. 2011;13(1):e30.

66. Lehne M, Sass J, Essenwanger A, Schepers J, Thun S. Why digital medicine depends on interoperability. NPJ Digit Med. 2019;2:79.

67. Ebert DD, Donkin L, Andersson G, et al. Does Internet-based guided-self-help for depression cause harm? An individual participant data meta-analysis on deterioration rates and its moderators in randomized controlled trials. Psychol Med. 2016;46(13):2679-2693.

68. Nicholas J, Larsen ME, Proudfoot J, Christensen H. Mobile Apps for Bipolar Disorder: A Systematic Review of Features and Content Quality. J Med Internet Res. 2015;17(8):e198.

69. Hawton K, Casanas ICC, Haw C, Saunders K. Risk factors for suicide in individuals with depression: a systematic review. J Affect Disord. 2013;147(1-3):17-28.

70. Huckvale K, Torous J, Larsen ME. Assessment of the Data Sharing and Privacy Practices of Smartphone Apps for Depression and Smoking Cessation. JAMA Netw Open. 2019;2(4):e192542.

71. The Joint Commission. Advancing Effective Communication, Cultural Competence, and Patientand Family-Centered Care: A Roadmap for Hospital. Oakbrook Terrace, IL: The Joint Commission;2010.

72. U.S. Department of Health and Human Services Office of Minority Health. The National CLAS Standards. U.S. Department of Health and Human Services Office of Minority Health. https://minorityhealth.hhs.gov/omh/browse.aspx?lvl=2\&lvlid=53. Published 2018. Accessed2020.

73. Smith A. U.S. Smartphone Use in 2015. Pew Research Center: Internet, Science \& Tech2015.

74. The Path Forward Intiative. The path forward for mental health and substance use: Improving access to effective treatment. Center for Workplace Health. http://workplacementalhealth.org/getmedia/37d2b0d2-016b-42b0-9a419e0debd7da7f/BH PF Action_Plan Executive Summary FINAL 1219. Published 2019. Accessed June 10, 2020, 2020.

75. Carlo AD, Drake L, Ratzliff ADH, Chang D, Unutzer J. Sustaining the Collaborative Care Model (CoCM): Billing Newly Available CoCM CPT Codes in an Academic Primary Care System. Psychiatr Serv. 2020:appips201900581. 
76. Counts N. Realizing the promise of digital therapeutics in mental health and substance use. Mental Health America.

https://mhanational.org/sites/default/files/MHA\%20Digital\%20Therapeutics\%20Issue $\% 20 \mathrm{Brief} \%$ 20FINAL.pdf. Published 2020. Accessed June 22, 2020, 2020. 